\title{
New concept for uvula manipulation to treat puberphonia
}

\begin{abstract}
Speech organs or articulators produce the sounds of language. The organs used for speech include the lips, teeth, alveolar bridge, hard palate, velum (soft palate), uvula, glottis, nasal cavity, pharynx and various parts of tongue. The vocal fold, in the larynx vibrates creating fluctuation in air pressure known as sound waves. The resonators of the mouth create formant regions and so different qualities sonorant (voiced) sound. We have a large series of vocal track vibrating endoscopic movie bank. We have created by treating 600 cases of puberphonia. We are able to record the change in the shape of the vocal tract and vibrating power of the airflow in the vocal tract. We are able to deduct and distinguish the different shape of the uvula and how airflow directed to the mouth by uvula. We are doing only uvula manipulation in treating puberphonia which gives immediate and persistent result. We are trying to bring out the importance of uvula in speech productions. The study concludes that the pharyngeal/uvula resonance manipulation therapy may be considered as an essential mode of treatment for puberphonia. Yet we like to inform that uvula is the structure in our body so little understood, so often abused and maligned.
\end{abstract}

Keywords: puberphonia, pharyngeal/uvula resonance manipulation, voice care, uvula, vocal tract, sound articulators, sound energy to the expiration air

\section{Introduction}

Speech organs or articulators produce the sound and delivered outside. It is recognized by language by the needy. Organs of speech constitute glottis, vellum (soft palate), hard palate, various parts of the tongue, alveolar bridge, teeth and lips. The structure in the larynx vibrates, creating fluctuation in the air pressure known as sound waves. The treatment with voice therapy or surgery to change the length, size and tension of the vocal cords to treat puberphonia continues to be discussed and debated by researchers and clinicians. ${ }^{1-3}$ This research work will provide an update include information on the logic, theory and evidence related to pharyngeal resonance manipulation therapy ${ }^{4}$ may be one of the important modality of treatment for puberphonia. The manipulation is done for change in pitch of the voice in the uvula. Incidentally we are able to through some light on the movement of the sound filled air travel from laryngopharynx to the oropharynx. We are able to study some of changes that happen in the uvula and the different air pressure changes in the phonatory apparatus.

\section{Objective}

Voice therapy for puberphonia is a promising modality of treatment but take long time for $78.9 \%$ success in 3 to 6 month time. ${ }^{5}$ Vocal cords manipulation / vocal cord crushed with the help of laryngeal forceps/ under GA with $83.33 \%$ success. ${ }^{6}$ Types 3 thyroplasty surgery tried in failed voice therapy. ${ }^{7}$ Our new pharyngeal resonance manipulation $^{8}$ in the treatment of puberphonia is similar to voice therapy. Instead of external voice therapy or external conventional laryngeal manipulation, we do internal pharyngeal manipulation at uvula. As internal pharyngeal resonance procedure needs no surgery it is cost effective and no post treatment complication. The surgeon who performs the procedure and the patient recognize the desired voice immediately.

\section{Methods and meterial}

The article is to give one another new easy way of immediate and permanent treatment for puberphonia and emphasizing the importance of uvula in speech production. We build the confidence for treating and ailing puberphonia clients. These are achieved by foster identification by voice pitch analyzer, effective one day treatment by uvula resonance manipulation, clients happy with getting the ancestral voice ${ }^{9}$ without any interventions in the vocal cord. As the treated patients are getting that ancestral voice habituation is easy and permeant. We have identified and treated 600 cases since 1990 to till date $13 / 03 / 2020$. The age of the patient is from 14 to 54 . With voice pitch analyzer we are able to identify the level of frequency in their voice. We build up the confidence of the patient by confirming their problem. We do complete ENT examination including endoscope of the vocal tract. All the patients had normal anatomy of the vocal tract. We had a one case of tracheostomy done following type 3 thyroplasty. This patient never had normal voice. We had 8 cases of bifid uvula. It is almost similar to the report given by various authors. ${ }^{10}$ Bifid uvula is nothing to do with puberphonia. ${ }^{11}$ This case also included in our study. All the patient reported are healthy. No other general problems. All the 600 cases reported only by referral by social media (Figure 1) (Tables 1\&2).

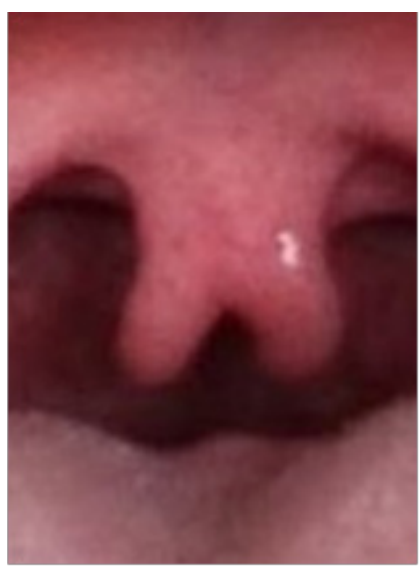

Figure I Puberphonia. 
Table I Total number of cases age, period of s

\section{Number of Patients 600}

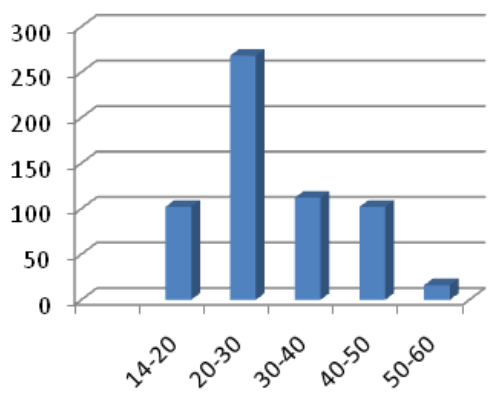

Number of patients

\begin{tabular}{llllll}
\hline Age & $\mathbf{1 4 - 2 0}$ & $\mathbf{2 0 - 3 0}$ & $\mathbf{3 0 - 4 0}$ & $\mathbf{4 0 - 5 0}$ & $\mathbf{5 0 - 6 0}$ \\
\hline $\begin{array}{l}\text { Num of Patients } \\
\begin{array}{l}\text { Total Number of } \\
\text { Patients }\end{array}\end{array}$ & 102 & 268 & 112 & 102 & 16 \\
\end{tabular}

Table 2 Total Number of Cases We Treated (1990- 16-03-2020 till date)

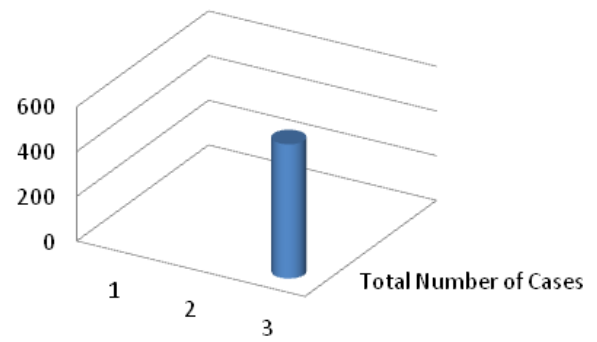

\begin{tabular}{|l|c|c|c|}
\cline { 2 - 4 } \multicolumn{1}{c|}{} & 1 & 2 & 3 \\
\hline$\square$ Total Number of Cases & & & 600 \\
\hline$\square$ & & & \\
\hline$\square$ & & & \\
\hline
\end{tabular}

\section{Discussion}

Importance of Uvula in articulation:

i) We humans may be one of the only vertebrates with this extra piece of tissue dangling off the back end of soft palate called uvula $^{12}$

ii) The uvula is an extension of the roof of mouth; what anatomists call the secondary palate. The function of the uvula seems pretty clear if we consider it as merely an extension of the soft palate. Galen (122-199 AD), one of the fathers of anatomy, believed that the uvula was important in speech and contributed to the beauty of the voice (Fritzell 1969) ${ }^{13}$

iii) While speaking the soft palate elevate and touches the roof of the nasopharynx. the air entry to the nose is prevented,

iv) If the soft palate cannot touch the back of the throat while speaking, air can enter the nasal cavity.

v) G.W. Back et al. directly observed the uvula in action using a flexible naso endoscope. During speech, they found that the uvula swings forward and forth while secreting saliva, essentially "basting" the throat, larynx, and vocal cords (Figures 2\&3). ${ }^{14}$

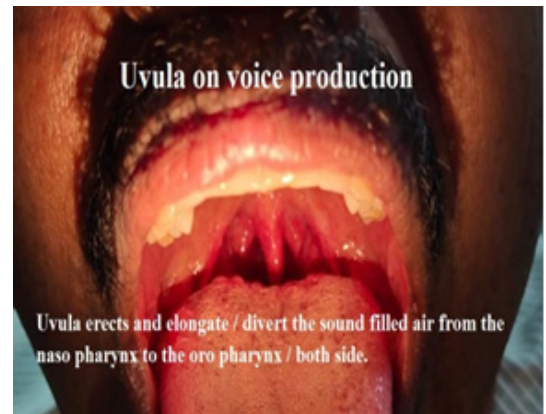

Figure 2 Uvula on voice production.

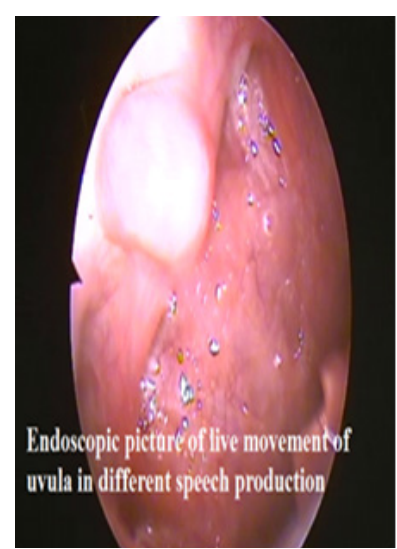

Figure 3 Endoscopic picture of live movement of uvula in different speech production.

Uvula come forward while air with sound energy enters from the laryngopharynx to the oropharynx and directs the same air on either side of the oral cavity. The velum or soft palate is seen in the diagram in a position that allows air to pass through the nose and through the mouth. The velum is probably in that position now, but often in speech it is raised so that air cannot escape through the nose. The other important thing about the velum is that it is one of the articulators that can be touched by the tongue (Figures $4 \& 5$ ). To produce speech sounds and speech requires air source. Sound waves need moving air, when we are speaking; the phase of breathing out is significantly longer, depending on the length of the word we want to produce. More gradual and more controlled speech, provide a regular flow of air which can be modified by the articulators in various ways.

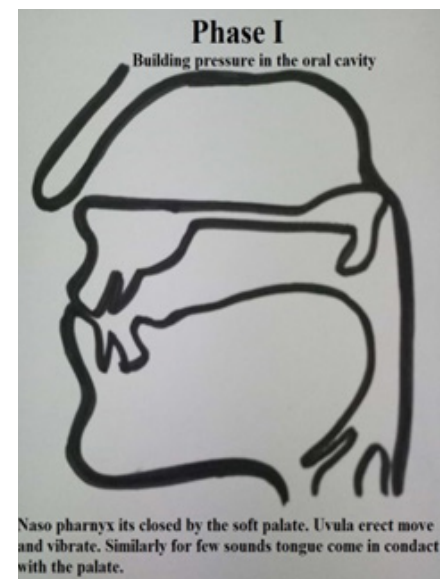

Figure 4 Uvula on phonation. 


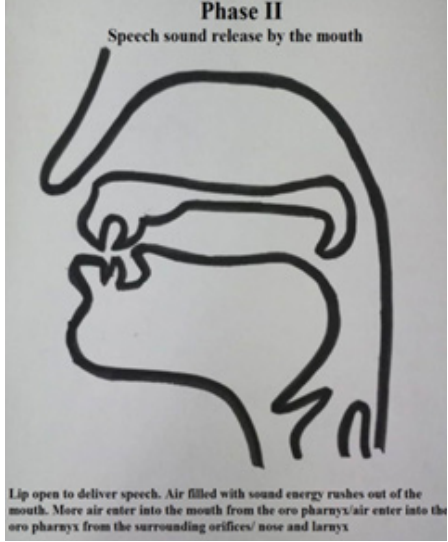

Figure 5 Uvula on normal breathing.

If we try to breath out and speak out at the same time the pitch of voice raises. The first is the glottic air stream mechanism, initiated by the movements of the vocal cords. It gives sound energy to the expiration air. The speech is produced in three levels as A, U,M mostly which can be modified on its way, through the mouth. We can feel the "A" sound at the pharynx (Level of uvula) "U" sound at the level of palate " $\mathrm{M}$ " sound at the level of buccal cavity. ${ }^{15}$ The air stream may be ingressive to produce implosive speech or aggressive in produces ejective speech. Sound filled air enters the mouth from the pharynx. It tries to attract more air from the nearby orifices in the pharynx (Figures 6-8).

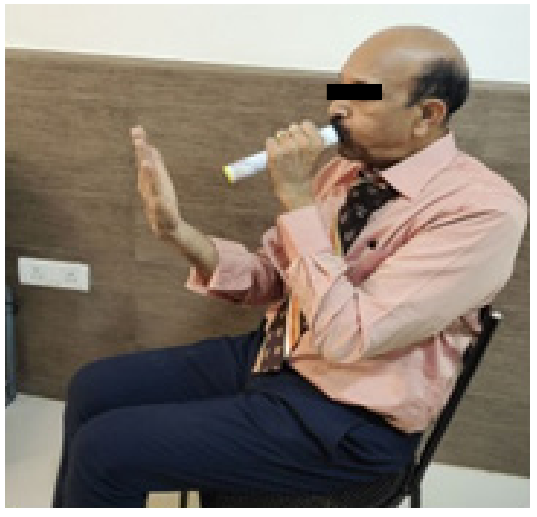

Figure 6 Air flow with limited force in a closed chamber.

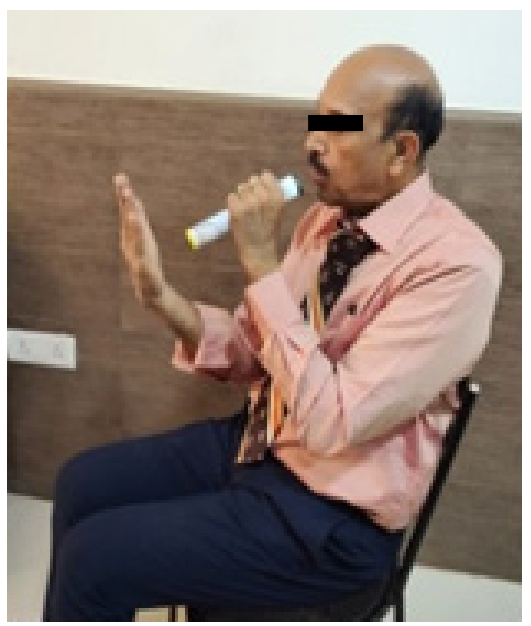

Figure 7 Air flow is abundance is forceful in open space (Pharynx).

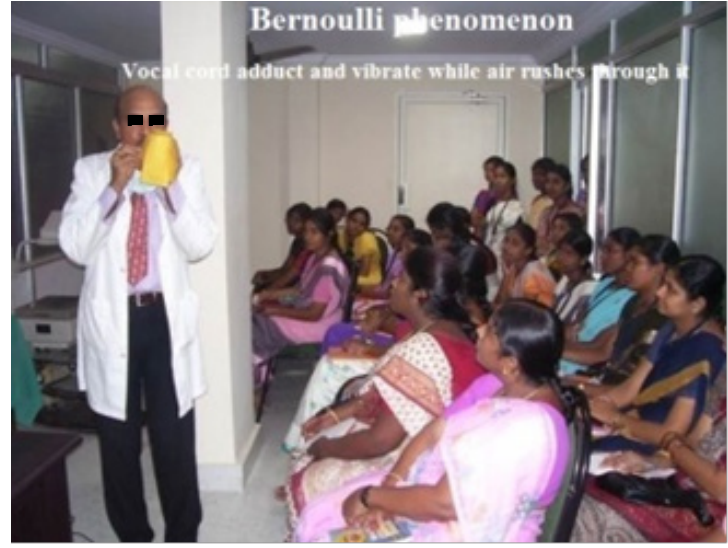

Figure 8 Vocal cord vibration produces and creates sound energy in the moving air (Bernoulli's phenomenon).

1. Air flow with limited force in a closed chamber

2. Air flow is abundance is forceful in open space (Pharynx).

The sound created at vocal cords float in the air/sound with air enters the pharynx. It is converted to speech sound by the mouth speech articulating resonating system. When we breathe out, the sound filled air travels up from laryngopharynx to oropharynx, powerful air flow is created in the direction of the air flow to buccal cavity. Uvula divides the air flow, right and left.

A. Nasal air also attracted towards the buccal air flow.

B. The divided air in the buccal cavity circulates and makes a powerful air flow.

C. The mouth shapes the sound to our voice speech pattern.

D. While opening the mouth the speech sounds are pushed out (Figure 9).

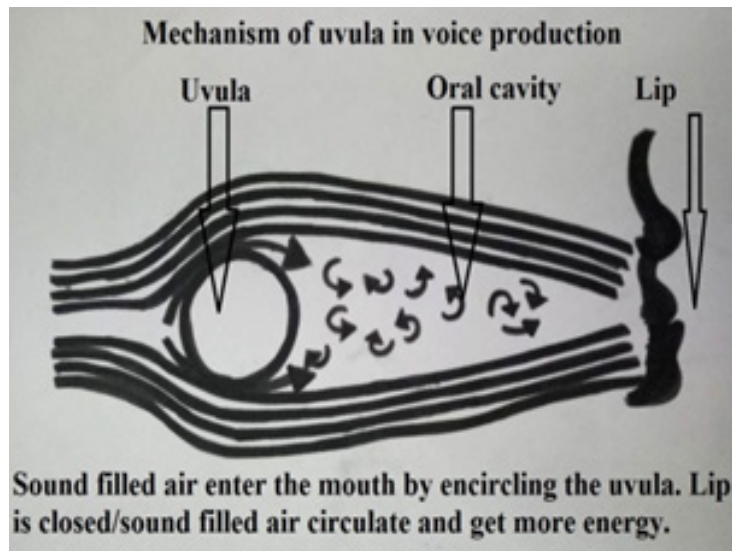

Figure 9 Mechanism of uvula in voice production.

On its way to mouth the sound filled air is divided to right and left by uvula. To increase its strength automatically it attracts air from the nasopharynx. The divided forceful air circulates in the mouth and increases the strength of air filled with sound. It gets the life of speech. Anything moves, it is life, vibrate and speech is produced (Figure 7).

Figure 10 On voice delivering lips open. The sound energy filled air comes out as speech. Air is pushed out on phonation. Pharynx gets the air from all the orifices and divert to the mouth (Figure 11). 


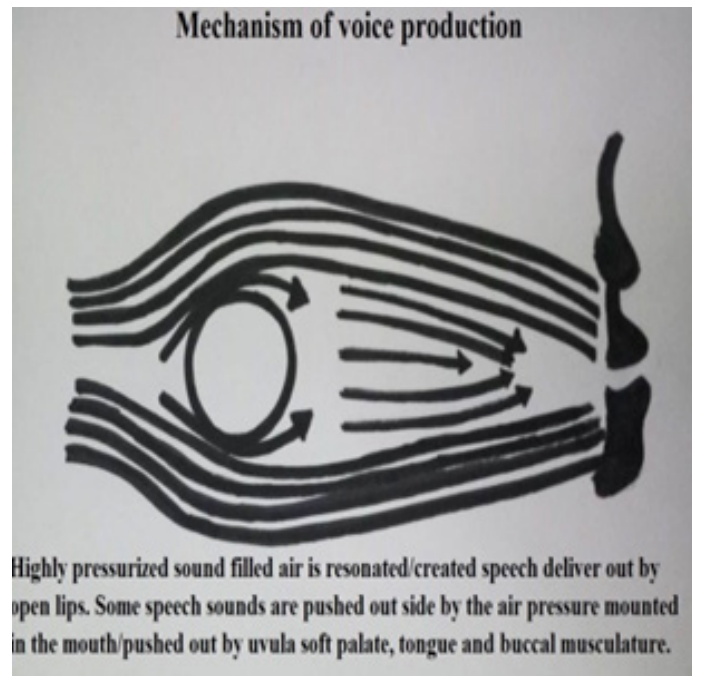

Figure 10 Mechanism of voice production.

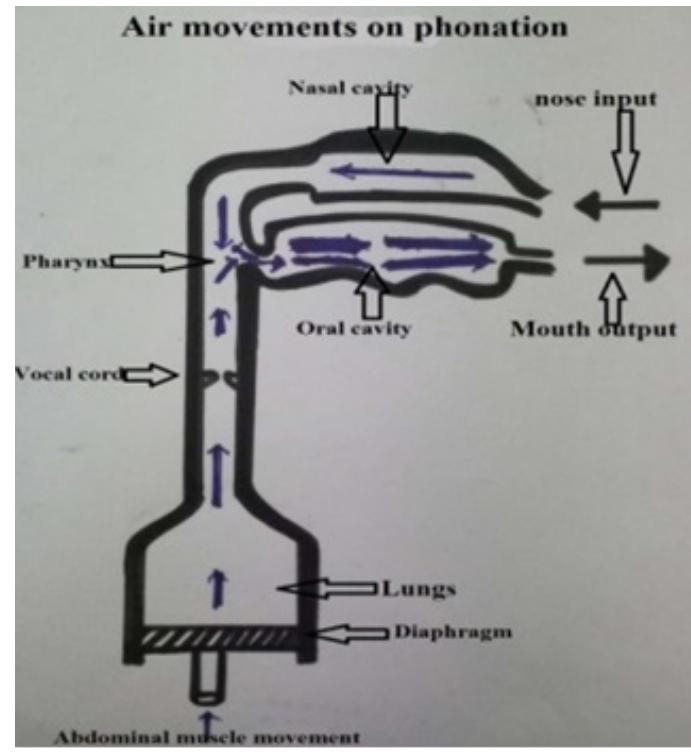

Figure I I Air flow during phonation.

\section{Conclusion}

The study concludes that the pharyngeal/uvula resonance manipulation therapy may be considered as an essential mode of treatment for puberphonia. Confident puberphonia treatment is required in the present time for the society. Its only bring awareness of puberphonia and the availability of puberphonia treatment because puberphonia treatment not only improves the voice, it is also improved the personality of puberphonia client and provides a thorough further knowledge about puberphonia among the society and health professionals. We observed similar to tongue movement, uvula also have specific movements. Yet we like to inform that uvula is the structure in our body so little understood, so often abused and maligned. Our work and suggestion is that uvula is not anatomical vignettes. It accomplishes several things:

a) The introduction of evolutionary concepts that uvula is a speech organ

b) Both uvula and speech serves to differentiate human beings from animals.
Uvula position, movement, secretion and action play a major role in augmenting the force of the air filled with sound, which is capable of producing more energy in voice production. Uvula moves vibrate increasing size and by its abundance salivary secretion keep the pharynx moist and well lubricated. Contraction of the musculature uvula add the bulk is reported by NabulA.Aggam(1977). Previous researches (eg.Lubker:Fritged) indicated the palate/ uvula does only on - off function in speech production. Lubta and Lindyvisegayffin .J(1970) suggested that palatal/uvula musculature would be predicted to contract more forcefully when necessary / to produce greater intra oral pressure and require for greater degree of oral tract construction required for voice production.

In this paper we present a description of uvula function during pharyngeal/uvula resonance manipulation for treating puberphonia. These observations are considered preliminary and are subject to revision as the data from all subjects are analyzed in great details with more refined methods.

\section{Recommendations}

I. The study can be done with ultra sound, magnetic resonance imaging study to assess the uvula size, shape and position in speech.

II. The measurement of air flow in the vocal tract (Appendix).

\section{Appendix}

Funding: This research received no specific grant from any funding agency, commercial or non profit sectors.

Ethical approval: All procedures performed in studies involving human participants were in accordance with the institutional research committee (USWR ethic approval) and with the 1964 Helsinki declaration and its later amendments.

Informed consent: Informed consent was obtained from all individual participants and their parents included in the study.

\section{Author contribution}

M.Kumaresan: Conceptualization of the study, collection, analysis of the data, writing the manuscript, finalizes the manuscript and will act as the guarantor of the paper; MK NB Conceptualization of the study, collection, analysis of the data, writing the manuscript, finalized the manuscript; MK: Edited and critically evaluated the manuscript.

\section{Acknowledgments}

I am immensely thankful to the SIVA ENT staffs that helped me for this manuscript development and function and also the volunteers who have done the heartfelt help for the research.

\section{Conflicts of interest}

Author declares that they have no conflict of interest.

\section{Funding}

None.

\section{References}

1. Desai V, Mishra P. Voice therapy outcome in puberphonia. J Laryngol Voice. 2012;2(1):26-29.

2. Vaidya S, Vyas G. Puberphonia: A novel approach to treatment. Indian J Otolaryngol Head Neck Surg. 2006;58(1):20-21. 
3. Khan MA, Khan M, Ahmed A, et al. Effectiveness of surgical technique of larynx manipulation in cases of puberphonia. J Postgrad Med Inst. 2019;33(1):57-59.

4. Kumaresan Muthiah, Navin Bharath Kumaresan. Assess the Impact of Puberphonia in the Society. International Journal of Otorhinolaryngology. 2019;5(2):39-43

5. ASR De Alwis, RJS Rupasinghe, Kumarasinghe I, et al. Efficacy of voice therapy in patients with puberphonia- a 15-year experience. Ceylon Journal of Otolaryngology. 2018.

6. Virginia M. Talmi Y, Zohar Y, et al. An overlooked factor in susceptibility to the common cold 1934. Readaptation of the velopharyngeal valve following the uvulopalatopharyngoplasty operation. Plastic and Reconstructive Surgery. 1988;82(1):20-27.

7. Kanishka Chowdhury, Somnath Saha, et al. Effects of Type 3 Thyroplasty on Voice Quality Outcomes in Puberphonia Philippine Journal of Otolaryngology Head and Neck Surgery. 2014;29(1).

8. Muhammad Ahmed Khan, Muhammad Khan, Attique Ahmed, et al. Effectiveness of surgical technique of larynx manipulation in cases of puberphonia. Journal of Postgraduate Medical Institute. 2019;33(1):6163.
9. Kumaresan M, Navin Bharath. Fast Track Treatment for Puberphonia. Scholarly Journal of Otolaryngology. 2020.

10. Azzam NA, Kuehn DP. The morphology of musculus uvulae. Pub Med. 1977;14(4):331.

11. Finkelstein Y, Meshorer A, Talmi YP, et al. The riddle of the uvula Otolaryngology - Head and Neck Surgery. 1992;107(3):444-450.

12. David M Darda. Central Washington University, Bringing Evolution into Anatomy and Physiology: The Uvula and the Story It Tells.

13. Fritzell B. The velopharyngel muscles in speech. Acta Otolaryngol Suppl. 1969. p. 250 .

14. Back GW, Nadig S, Uppal S. Coatesworth AP, Why do we have a uvula?: Literature review and a new theory. Clinical Otolaryngology. 2004;29(6):689-693.

15. Lahri RK. Aum- The sphotachandogyaupanishal 2005. p. 1-2. 